\title{
Mesenchymal Stem Cell-Derived Extracellular Vesicles: A Novel Cell-Free Therapy for Sepsis
}

\author{
Yanwei Cheng ${ }^{1 \dagger}$, Xue $\mathrm{CaO}^{2 \dagger}$ and Lijie Qin ${ }^{1 *}$ \\ 1 Department of Emergency, Henan Provincial People's Hospital, People's Hospital of Zhengzhou University, People's \\ Hospital of Henan University, Zhengzhou, China, ${ }^{2}$ Department of Rheumatology and Immunology, Henan Provincial People's \\ Hospital, People's Hospital of Zhengzhou University, People's Hospital of Henan University, Zhengzhou, China
}

\section{OPEN ACCESS}

Edited by:

Lukas Martin

University Hospital RWTH

Aachen, Germany

Reviewed by:

Sebastian Wendt,

University Hospital RWTH

Aachen, Germany

Yasser Mohamed El-Sherbiny, Nottingham Trent University,

United Kingdom

${ }^{*}$ Correspondence:

Lijie Qin

qinlijie1819@163.com

${ }^{\dagger}$ These authors share first authorship

Specialty section:

This article was submitted to Inflammation,

a section of the journal

Frontiers in Immunology

Received: 12 January 2020

Accepted: 23 March 2020

Published: 21 April 2020

Citation:

Cheng Y, Cao X and Qin L (2020)

Mesenchymal Stem Cell-Derived Extracellular Vesicles: A Novel

Cell-Free Therapy for Sepsis.

Front. Immunol. 11:647.

doi: 10.3389/fimmu.2020.00647
Sepsis remains a serious and life-threatening disease with high morbidity and mortality. Due to the unique immunomodulatory, anti-inflammatory, anti-apoptotic, anti-microbial, anti-oxidative, and reparative properties, mesenchymal stem cells (MSCs) have been extensively used in preclinical and clinical trials for diverse diseases and have shown great therapeutic potential in sepsis. However, concerns remain regarding whether MSCs can become tumorigenic or have other side effects. Extracellular vesicles (EVs) are a heterogeneous group of membrane-enclosed particles released from almost any cell and perform an important role in intercellular communication. Recently, it has emerged that EVs derived from MSCs (MSC-EVs) appear to exert a therapeutic benefit similar to MSCs in protecting against sepsis-induced organ dysfunction by delivering a cargo that includes RNAs and proteins to target cells. More importantly, compared to their parent cells, MSC-EVs have a superior safety profile, can be safely stored without losing function, and possess other advantages. Hence, MSC-EVs may be used as a novel alternative to MSC-based therapy in sepsis. Here, we summarize the properties and applications of MSC-EVs in sepsis.

Keywords: mesenchymal stem cell-derived extracellular vesicles (MSC-EVs), sepsis-induced acute lung injury, sepsis-induced acute kidney injury, sepsis-associated cardiovascular disorder, sepsis-induced liver injury

\section{INTRODUCTION}

In the recent "sepsis-3.0" consensus (1), sepsis is defined as a life-threatening, multiorgan dysfunction caused by a dysregulated host response to infection. In view of the high morbidity and mortality, sepsis has been described as the quintessential medical disorder of the 21 st century. Despite continuous progress in the development of therapeutic strategies, sepsis is still a major clinical problem and remains the leading cause of death $(2,3)$ in the critically ill patient population, due to uncontrolled inflammation together with immunosuppression. In such a context, an investigation into novel therapies to ameliorate sepsis would be urgently needed.

With the emergence of stem cells as potential therapeutic agents for diverse diseases, attempts to use stem cells, especially mesenchymal stem cells (MSCs), to ameliorate sepsis in animal models have increased exponentially. Growing preclinical data have suggested that MSCs can directly modify pathophysiology and underlying injury mechanisms in sepsis through the immunomodulatory, anti-bacterial, anti-inflammatory, anti-oxidative, anti-apoptotic, and reparative properties. It is worth noting that MSCs have also been confirmed as having a therapeutic effect on sepsis in two clinical trials $(4,5)$. Even then, the utilization of MSCs as therapeutic 
agents for sepsis has raised several concerns over the past decade, including senescence-induced genetic instability, the heterogeneity of MSCs populations, quality assurance challenges of MSCs, and finding optimal MSCs tissue sources. Most crucially, the significant hurdles that potential therapeutics face in the future are MSCs safety concerns. It has been reported that MSCs play a dual role in immune regulation; on the one hand, MSCs exhibit immunosuppressive function (6), while on the other hand, MSCs may act as antigen-presenting cells, inducing an immune response (7). In addition, MSCs can potentially become tumorigenic either by direct malignant transformation of MSCs or indirectly by facilitating the growth of other tumor cells (8). So far MSCs have been found in several tumor types, including gastric adenocarcinoma (9), lipoma (10), and osteosarcoma (11), strongly indicating their involvement in tumor development. In light of this fact, the probability of the risks must be cautiously weighed against the potential benefits to every patient.

Recently, special attention has been paid to the extracellular vesicles (EVs) derived from MSCs (MSCs-EVs), which can function as shuttles for the delivery of a cargo that includes RNAs and proteins from parental to target cells and are as biologically active as the parent cells themselves (12-15). Hence, it is conceivable that the protective effect of MSCs on sepsis is at least partly due to the release of EVs.

\section{CHARACTERIZATION OF MSC-EVS (FIGURE 1)}

EVs are a heterogeneous group of membrane-enclosed particles that can be released from almost any cell. Based on the current knowledge of their size and biogenesis, EVs can be classified into three broad groups: exosomes (Exos), microvesicles (MVs), and apoptotic bodies. Exos $(40-150 \mathrm{~nm})$ bud from the endosomal system, whereas MVs $(100-1,000 \mathrm{~nm})$ are shed directly from the plasma membrane, and apoptotic bodies (1,000-5,000 nm) originate from apoptotic cells (16-18). In general, apoptotic bodies can be removed by the commonly used isolation method of ultracentrifugation (19). It should be noted that each individual cell can release both Exos and MVs simultaneously. However, so far it is impossible to unanimously distinguish MVs and Exos. In addition, most studies have not clearly defined the origin of EVs under study. Therefore, we here use EVs as an umbrella term to include both MVs and Exos.

MSC-EVs are released from resting MSCs and can be markedly increased during cellular activation or cell stress $(12,20,21)$. They contain a trove of bioactive substances such as proteins, lipids, and most importantly, nucleic acids, thereby playing an important role in immune modulation, proangiogenesis, anti-apoptosis, and tissue regeneration. Up to now, over 5,000 species of MSC-EVs proteins have been characterized, including mediators controlling self-renewal, differentiation, signal transduction, and additional MSCs antigens affecting the migration of MSCs $(19,22)$. In addition, MSC-EVs contain a number of adhesion molecules such as CD44, CD29, alpha 4 -integrin, and alpha 5-integrin etc., which contribute to the identification of MSC-EVs (22). Accumulating evidence has demonstrated that MSC-EVs modulate immune response by producing cytokines, growth factors, and tolerogenic molecules, such as IL-10, IL-6, IL-37, and lipocalin-2 and transforming growth factor (TGF)- $\beta$, programmed death ligand-1(PD-L1), galectin-1, etc. $(20,23,24)$. Other than proteins, MSC-EVs are enriched with nucleic acids, including mRNA, microRNA (miRNA), and DNA (25). It has been demonstrated that the transfer of miRNAs from MSC-EVs to target cells may be the underlying mechanism in alleviating injury of the kidney $(26,27)$, heart (28), liver (29), and brain (30). In recent years, more than 150 miRNAs have been identified in the cargo of MSC-EVs. These miRNAs are usually related to apoptosis, tumorigenesis, immune responses, angiogenesis, and organism development, such as miR-221, miR-23b, miR-125b, miR-451, miR-31, miR-24, miR-214, miR-122, miR-16, miR-150, and miR133b, etc. $(19,31-34)$. All the internal components in MSC-EVs are surrounded by a bilayer of lipids that play an important role in protecting them. Compared to their parent cells, EVs are enriched with some specific lipids (cholesterol, glycosphingolipids, and phosphatidylserine) (35-38), making them more functional. Our previous study (21) also showed that sphingosine-1-phosphate enriched within MSC-EVs could enhance the repair effect of MSCs for articular cartilage.

\section{THERAPEUTIC APPLICATIONS OF MSC-EVS IN SEPSIS}

Through delivering proteins and nucleic acids, MSC-EVs seem to hold many functions of the MSCs themselves and are described as a new mechanism of intercellular communication (39). Nowadays, increasing studies have clearly confirmed that EVs alone are responsible for the therapeutic effects of MSCs in sepsisinduced organ dysfunction, including sepsis-induced acute lung injury, sepsis-induced acute kidney injury, sepsis-associated cardiovascular disorder, and sepsis-induced liver injury (40). Accordingly, MSCs-EVs may be an alternative therapy to MSCs and will be the next-generation therapeutic route for sepsis.

\section{MSC-EVs and Sepsis-Induced Actue Lung Injury}

Acute lung injury (ALI) is the most common organ injury in septic patients and leads to a greater mortality. In the past few years, the beneficial effects of MSCs on sepsis-induced ALI have been shown to be attributed to the release of EVs. Using LPSinduced ALI in an ex vivo perfused human lung, Park et al. (41) reported that MSC-EVs significantly increased alveolar fluid clearance and reduced protein permeability and numerically lowered the bacterial load in the injured alveolus. In a mouse model of E. coli endotoxin-induced ALI, Zhu et al. (42) also confirmed the similar beneficial effects of human MSC-EVs and further demonstrated that the protection was in part due to the delivery of keratinocyte growth factor (KGF) mRNA from MSCEVs to the injured alveolar epithelium and lung endothelium. This falls in good accordance with Monsel's study (43) that MSCEVs improved survival in ALI from E. coli pneumonia via a 


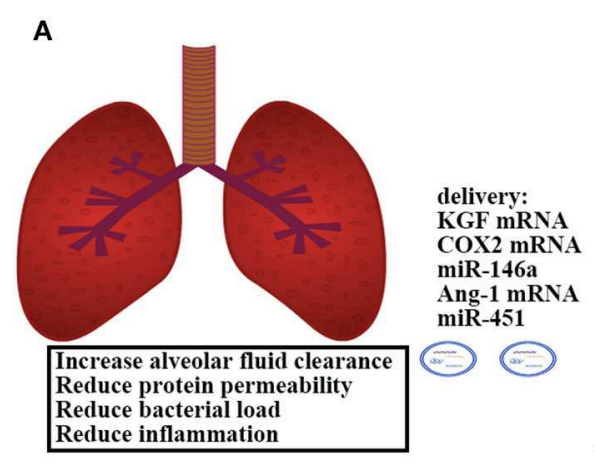

C
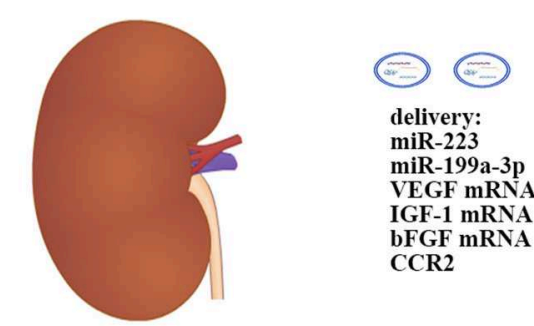

Enhance angiogenesis

Reduce tubular cell apoptosis

Reduce pro-inflammatory cytokines

Restore anti-inflammatory cytokines
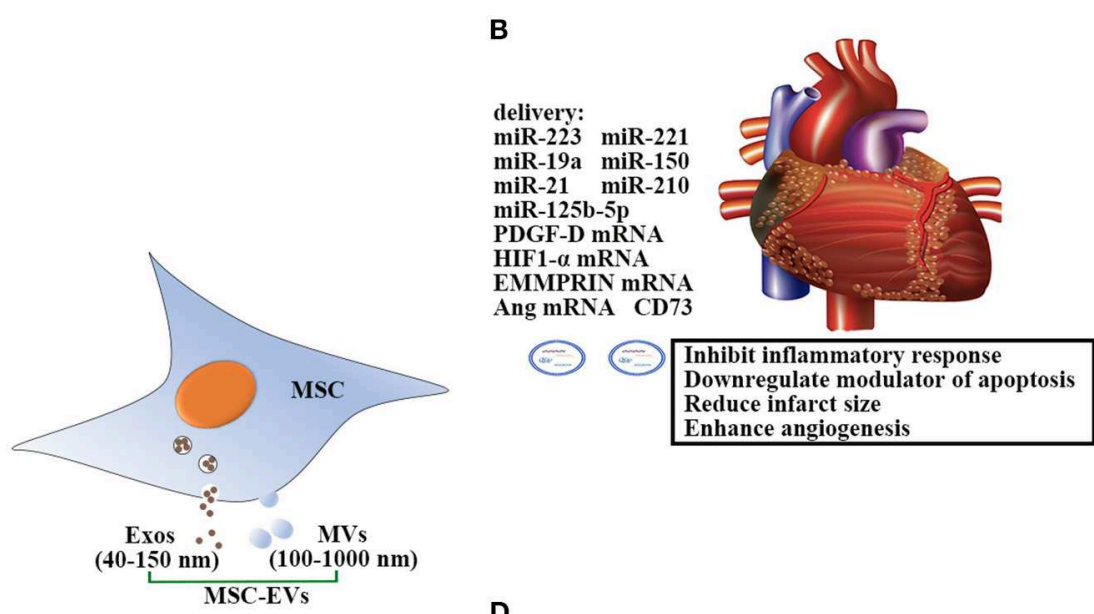

D

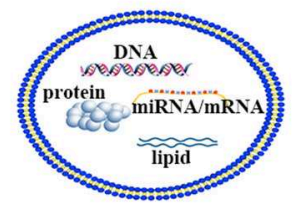

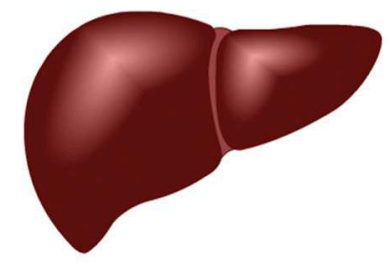

FIGURE 1 | Therapeutic effects and mechanisms of the action of MSC-EVs in: (A) sepsis-induced acute lung injury; (B) sepsis-induced acute kidney injury; (C) sepsis-associated cardiovascular disorder; (D) sepsis-induced liver injury. EVs comprise a heterogeneous population of membrane vesicles of various origins. Here we use EVs as an umbrella term to include both MVs and Exos. MSC-EVs can shuttle various cargoes, including proteins, lipids, and nucleic acids, to the recipient cells to protect against sepsis-induced organ dysfunction. Ang, Angiopoietin; bFGF, Basic fibroblast growth factor; COX2, cyclooxygenase 2; CCR2, C-C motif chemokine receptor-2; CXCL2, Chemokine Ligand 2; EVs, Extracellular vesicles; Exos, Exosomes; EMMPRIN, Extracellular matrix metalloproteinase inducer; GPX1, Glutathione peroxidase 1; HIF1- $\alpha$, Hypoxia-inducible factor 1-alpha; HGF, Hepatocyte growth factor; IL6ST, Interleukin 6 signal transducer; IGF-1, Insulin-like growth factor 1; KGF, Keratinocyte growth factor; MVs, Microvesicles; MSCs, Mesenchymal Stem Cells; miR, microRNA; PDGF-D, Platelet-derived growth factor D; VEGF, Vascular endothelial growth factor.

mechanism partially dependent on KGF secretion. The authors (43) further discovered that MSC-EVs could enhance monocyte phagocytosis of bacteria through transferring cyclooxygenase 2 (COX2) mRNA to monocytes, with a resultant increase in prostaglandin E2 (PGE2) secretion. PGE2 is essential for transforming the polarization of monocytes-macrophages M1 into M2 phenotype (44-46). In addition, Song et al. (47) showed that miR-146a promoted polarization of M2 macrophages and finally led to increased survival in septic mice. MSC-EVs also contain a substantial quantity of angiopoietin-1 (Ang-1) mRNA, which plays an essential role in vascular stabilization and resolving inflammation. In the current study, Tang et al. (48) demonstrated that the therapeutic benefit of EVs in ALI, and their immunomodulatory properties on macrophages, were partly mediated through their content of Ang-1 mRNA. Furthermore, the transfer of miR-451 and mitochondria from MSC-EVs to the alveolar epithelium and macrophages also contributed to preventing ALI (49). Altogether, these data indicate that
MSC-EVs may represent a novel therapeutic option for sepsisinduced ALI.

\section{MSC-EVs and Sepsis-Induced Actue Kidney Injury}

Mounting studies have provided convincing evidence that MSCs exert their renoprotective effects by releasing EVs in several acute kidney injury (AKI) models. AKI is commonly present in more than $20 \%$ of septic patients (50). However, data about the therapeutic effects of MSC-EVs in sepsis-induced AKI are scarce. Currently, the anti-apoptosis (51), pro-angiogenesis (52), and anti-inflammatory (53) properties of MSC-EVs have been considered as the important mechanistic approaches to ameliorate AKI induced by different causes. In AKI mice models induced by ischemia-reperfusion injury, Bruno et al. (27) found that MSC-EVs induced the expression of anti-apoptotic genes (Bcl-XL, Bcl2, and BIRC8) in renal tubular epithelial cells while simultaneously down-regulating pro-apoptotic genes 
(Casp1, Casp8, and LTA), thus reducing tubular cell apoptosis and conferring an anti-apoptotic phenotype necessary for tissue repair. Interestingly, the anti-apoptosis effect of MSC-EVs may be mediated in part by the transfer of miRNAs (miR-223 and miR-199a-3p) to tubular cells $(54,55)$. In addition, MSCEVs can directly shuttle several pro-angiogenesis transcription factors including vascular endothelial growth factor (VEGF) (52), insulin-like growth factor 1 (IGF-1) (56), and basic fibroblast growth factor (bFGF) (57) to the damaged renal tubular epithelial cells and enhance angiogenesis, which is considered as an important step in tissue regeneration. In a mouse model of AKI induced by glycerol, Bruno (58) demonstrated that $15 \mu \mathrm{g}$ of MSC-EVs injected intravenously activated the proliferative progress in viable tubular cells through their pro-angiogenesis effects. Importantly, the effects of MSC-EVs on the recovery of AKI were as effective as their parental MSCs. It is well known that excessive inflammation is the culprit leading to sepsis-induced AKI. MSC-EV s can modulate renal inflammation by reducing the release of several pro-inflammatory cytokines, including IL-1, IL6 , and TNF- $\alpha$, while restoring the level of the anti-inflammatory cytokine IL-10 $(26,53,59)$. This anti-inflammatory effect may be associated with the $\mathrm{C}-\mathrm{C}$ motif chemokine receptor-2 (CCR2) contained within MSC-EVs, which can suppress macrophages function and attenuate renal injury (60). At present, MSC-EVs have been studied in patients with chronic kidney disease (61). The results showed that administration of MSC-EVs had no side effects and caused significant improvement of the kidney function. Collectively, there are good reasons to believe that MSC-EVs could be exploited as a new therapeutic approach for sepsis-induced AKI.

\section{MSC-EVs and Sepsis-Associated Cardiovascular Disorder}

Despite only a few studies having addressed potential cardioprotective effects of MSCs on sepsis-induced cardiovascular disease, the data still support that MSCs mediate their protection through the secretion of EVs. In septic mice induced by cecal ligation and puncture (CLP), MSC-EVs displayed cardioprotective benefits and enhanced survival of cardiomyocyte cells. In this research, Wang et al. (28) further proved that miR-223 enriched in MSC-EVs was critical for EVselicited action in sepsis. miR-223 can inhibit the expression of Sema3A and Stat3, negatively regulating the expression of many inflammatory genes, such as TNF- $\alpha$, IL-6, and IL-1 (62). Another important piece of research by Tabet et al. (63) also mentioned that miR-223 was able to alleviate the inflammatory process in human coronary artery endothelial cells by downregulating intercellular adhesion molecule 1 . In addition, some antiapoptotic miRNAs contained within MSCs-EVs showed a vital role in cardioprotection. For example, miR-221 can reduce the expression of p53 upregulated modulator of apoptosis and is more highly enriched in MSC-EVs than that in their parent MSCs. Numerous studies $(34,64)$ have confirmed that the delivery of miR-221 contained within EVs contributed to the cardioprotection by MSC-EVs. A study by Yu et al. (65) demonstrated that MSC-EVs enriched with anti-apoptotic
miR-19a also restored cardiac function and reduced infarct size in a rat model of acute myocardial infarction (AMI). In addition to miRNAs, the protein of CD73 on the surface of MSC-EVs was suggested to overcome the pro-apoptotic milieu of the perfused myocardium (66). In AMI mice models, Bian et al. (67) reported that intramyocardial injection of MSC-EVs markedly promoted angiogenesis and reduced infarct size. They further identified that MSC-EVs might be a mixture of miR-150-enriched MVs and Exos (33). Moreover, the pro-angiogenesis effect of the other cargo of MSC-EVs had been investigated in vitro and in vivo MI models, including miR-210 (66), miR-21 (68), platelet-derived growth factor D (PDGF-D) (69), hypoxia-inducible factor 1alpha (HIF1- $\alpha)$ (70), and extracellular matrix metalloproteinase inducer (EMMPRIN) (71) and angiopoietin (72). Recently, Xiao et al. (73) demonstrated that the beneficial effects offered by MSCs in MI model were at least partially because of improved autophagic flux through excreted EVs containing mainly miR125b-5p. On the basis of these studies, MSC-EVs seem to be an ideal therapeutic agent for sepsis-induced cardiovascular disease in the near future.

\section{MSC-EVs and Sepsis-Induced Liver Injury}

During sepsis, liver injury occurs frequently and contributes to the pathogenesis of multiple organ dysfunction, and has been considered as an early indicator of poor outcome in septic patients $(74,75)$. Studies in a range of liver disease models demonstrated that MSC-EVs could suppress inflammatory response, reduce hepatocyte apoptosis, and enhance liver regeneration. However, few applications of MSC-EVs into sepsisinduced liver injury are available. Nong et al. (76) studied the hepatoprotective effect of MSC-EVs in a mouse model of hepatic ischemia-reperfusion injury. They showed that human-induced pluripotent stem cell-derived MSC-EVs protected hepatocytes via reducing pro-inflammatory cytokine production, such as TNF- $\alpha$, IL-6, and high mobility group box 1 (HMGB1). Likewise using a concanavalin-A-induced liver injury model, Tamura et al. (77) demonstrated that MSC-EVs decreased production of proinflammatory cytokines, while anti-inflammatory cytokine and regulatory $\mathrm{T}$ cell levels increased. Recently, the anti-apoptotic and anti-oxidant effects of MSC-EVs were also investigated. A study in a lethal mouse model of liver failure induced by Dgalactosamine/TNF- $\alpha$ showed that MSC-EV s containing Y-RNA1 activated anti-apoptotic pathways, thus mitigating hepatic injury and enhancing survival (78). In carbon tetrachloride (CCl4)-induced liver injury, Yan et al. (79) demonstrated that MSC-EVs promoted the recovery of hepatic oxidant injury through the delivery of glutathione peroxidase 1 (GPX1). Additionally, Tan et al. (80) confirmed that MSC-EVs were capable of promoting hepatocyte regeneration by inducing the IL-6/STAT3 pathway and cell cycle progression. Proteins in MSCEVs, such as interleukin 6 signal transducer (IL6ST), chemokine Ligand 2 (CXCL2), and hepatocyte growth factor (HGF) etc., were found to be involved in the liver-regeneration process (80). However, it remains to be determined whether miRNAs or other mRNAs within MSC-EVs may have any impact on the proliferation effect of the hepatocytes. According to these studies, MSC-EVs may be considered as a novel therapeutic approach for 
alleviating sepsis-induced liver injury, and correlative research should fill the gap in this field in the future.

\section{ADVANTAGES AND CHALLENGES OF MSC-EVS THERAPY}

Compared to MSC-based therapy, MSC-EVs therapy offers more advantages. First, MSC-EVs are highly stable and suitable for long-term storage without the addition of potentially toxic cryopreservatives $(21,81,82)$. Second, MSC-EVs can induce stronger signaling in intercellular communication by directly transferring functional proteins and miRNAs to the recipient cells $(83,84)$. Third, MSC-EVs have no heterologous risk $(85,86)$ and no immune response after allogeneic application (87). Furthermore, a significant advantage of MSC-EVs is to circumvent the potential tumorigenicity of MSCs. So far, there is no evidence showing the oncogenic potential of MSC-EVs. A study (88) reported that MSC-EVs can inhibit tumor growth and exert an anti-tumor activity both in vitro and in vivo. Given this, MSC-EVs look to be a novel and promising therapeutic approach for sepsis.

However, there remain significant challenges that need to be addressed prior to the potential application of MSC-EVs in human sepsis. First, the protocol for isolation has to be standardized. Currently, no "gold standard protocol" for EVs isolation exists, instead different isolation methods are used to isolate different subsets of EVs even though the source is the same. Second, the suitable markers to analyze EVs at a single-vesicle level must be established. So far no consensus exists regarding the nomenclature of EVs or the markers that distinguish each type of EV once they have been secreted or shed from the cells. Third, there is a paucity of knowledge about the content within MSC-EVs. EVs contain a trove of cellular cargos, which are able to act as diverse functions and can vary widely between sources and conditions. It is unknown which effector molecules are important within EVs or which are not protective or harmful. Meanwhile, there is a critical need for exploiting possible ways to modify MSC-EVs composition

\section{REFERENCES}

1. Singer M, Deutschman CS, Seymour CW, Shankar-Hari M, Annane D, Bauer $\mathrm{M}$, et al. The Third International Consensus definitions for sepsis and septic shock (sepsis-3). JAMA. (2016) 315:801-10. doi: 10.1001/jama. 2016.0287

2. Melamed A, Sorvillo FJ. The burden of sepsis-associated mortality in the United States from 1999 to 2005: an analysis of multiple-cause-of-death data. Crit Care. (2009) 13:R28. doi: 10.1186/cc7733

3. Dombrovskiy VY, Martin AA, Sunderram J, Paz HL. Rapid increase in hospitalization and mortality rates for severe sepsis in the United States: a trend analysis from 1993 to 2003. Crit Care Med. (2007) 35:124450. doi: 10.1097/01.CCM.0000261890.41311.E9

4. Wilson JG, Liu KD, Zhuo H, Caballero L, McMillan M, Fang X, et al. Mesenchymal stem (stromal) cells for treatment of ARDS: a phase 1 clinical trial. Lancet Respir Med. (2015) 3:24-32. doi: 10.1016/S2213-2600(14)70291-7

5. Galstian GM, Parovichnikova EN, Makarova PM, Kuzmina LA, Troitskaya VV, Gemdzhian E, et al. The results of the russian clinical trial and modulate their biological activities. Fourth, the therapeutic capacity of MSC-EVs derived from different sources in sepsis should be investigated. In addition, much work is needed on how to optimize the methods to produce MSC-EVs on a large scale, how to validate the dosage and half-life of MSC-EVs, and confirming the potential effects of MSC-EVs in the late stage of sepsis. Finally, the unknown negative effects of MSC-EVs have to be clarified.

\section{CONCLUSIONS}

Extracellular vesicles (EVs) are naturally released from almost any cell and participate in cell-to-cell communication in physiological as well as pathological processes by transferring their components, such as proteins, miRNA, mRNA, and even mitochondria. A study (89) considered EVs as possible culprits during the pathogenesis of sepsis, whereas EVs derived from MSCs showed striking therapeutic benefits in sepsis. In this review, we have summarized the recent knowledge related to the therapeutic applications of MSC-EVs in sepsis. These considerable preclinical data support the hypothesis that cellfree therapy with MSC-EVs could be a novel alternative MSC-based therapy in sepsis, especially in early stage sepsis. However, realizing this promising therapeutic approach of MSCEVs would require extensive testing to validate their safety and efficacy.

\section{AUTHOR CONTRIBUTIONS}

YC and XC wrote the first draft of this article and designed the figure. LQ critically revised the manuscript for important intellectual content. All authors approved the final version.

\section{FUNDING}

The present work was supported by the 23456 Talent Project of Henan Provincial People's Hospital to LQ, Research Startup fund of Henan Provincial People's Hospital to YC and XC. of mesenchymal stromal cells (MSCs) in severe neutropenic patients (pts) with septic shock (SS) (RUMCESS trial). Blood. (2015) 126:2220. doi: 10.1182/blood.V126.23.2220

6. Jarvinen L, Badri L, Wettlaufer S, Ohtsuka T, Standiford TJ, Toews GB, et al. Lung resident mesenchymal stem cells isolated from human lung allografts inhibit T cell proliferation via a soluble mediator. J Immunol. (2008) 181:438996. doi: 10.4049/jimmunol.181.6.4389

7. Stagg J, Pommey S, Eliopoulos N, Galipeau J. Interferon-gamma-stimulated marrow stromal cells: a new type of nonhematopoietic antigen-presenting cell. Blood. (2006) 107:2570-7. doi: 10.1182/blood-2005-07-2793

8. Rosland GV, Svendsen A, Torsvik A, Sobala E, McCormack E, Immervoll H, et al. Long-term cultures of bone marrow-derived human mesenchymal stem cells frequently undergo spontaneous malignant transformation. Cancer Res. (2009) 69:5331-9. doi: 10.1158/0008-5472.CAN-08-4630

9. Xu X, Zhang X, Wang S, Qian $\mathrm{H}$, Zhu W, Cao H, et al. Isolation and comparison of mesenchymal stem-like cells from human gastric cancer and adjacent non-cancerous tissues. J Cancer Res Clin Oncol. (2011) 137:495504. doi: 10.1007/s00432-010-0908-6 
10. Lin TM, Chang HW, Wang $\mathrm{KH}$, Kao AP, Chang CC, Wen $\mathrm{CH}$, et al. Isolation and identification of mesenchymal stem cells from human lipoma tissue. Biochem Biophys Res Commun. (2007) 361:8839. doi: 10.1016/j.bbrc.2007.07.116

11. Brune JC, Tormin A, Johansson MC, Rissler P, Brosjo O, Lofvenberg R, et al. Mesenchymal stromal cells from primary osteosarcoma are non-malignant and strikingly similar to their bone marrow counterparts. Int J Cancer. (2011) 129:319-30. doi: 10.1002/ijc.25697

12. Tan X, Gong YZ, Wu P, Liao DF, Zheng XL. Mesenchymal stem cellderived microparticles: a promising therapeutic strategy. Int J Mol Sci. (2014) 15:14348-63. doi: 10.3390/ijms150814348

13. Carandini T, Colombo F, Finardi A, Casella G, Garzetti L, Verderio C, et al. Microvesicles: what is the role in multiple sclerosis? Front Neurol. (2015) 6:111. doi: 10.3389/fneur.2015.00111

14. Schneider A, Simons M. Exosomes: vesicular carriers for intercellular communication in neurodegenerative disorders. Cell Tissue Res. (2013) 352:33-47. doi: 10.1007/s00441-012-1428-2

15. Toh WS, Lai RC, Hui JHP, Lim SK. MSC exosome as a cell-free MSC therapy for cartilage regeneration: implications for osteoarthritis treatment. Semin Cell Dev Biol. (2017) 67:56-64. doi: 10.1016/j.semcdb.2016.11.008

16. Karpman D, Stahl AL, Arvidsson I. Extracellular vesicles in renal disease. Nat Rev Nephrol. (2017) 13:545-62. doi: 10.1038/nrneph.2017.98

17. Chaput N, Thery C. Exosomes: immune properties and potential clinical implementations. Semin Immunopathol. (2011) 33:41940. doi: 10.1007/s00281-010-0233-9

18. Thery C. Exosomes: secreted vesicles and intercellular communications. F1000 Biol Rep. (2011) 3:15. doi: 10.3410/B3-15

19. Jafarinia M, Alsahebfosoul F, Salehi H, Eskandari N, Ganjalikhani-Hakemi M. Mesenchymal stem cell-derived extracellular vesicles: a novel cell-free therapy. Immunol Invest. (2020). doi: 10.1080/08820139.2020.1712416. [Epub ahead of print].

20. Mokarizadeh A, Delirezh N, Morshedi A, Mosayebi G, Farshid AA, Mardani K. Microvesicles derived from mesenchymal stem cells: potent organelles for induction of tolerogenic signaling. Immunol Lett. (2012) 147:4754. doi: 10.1016/j.imlet.2012.06.001

21. Xiang C, Yang K, Liang Z, Wan Y, Cheng Y, Ma D, et al. Sphingosine-1phosphate mediates the therapeutic effects of bone marrow mesenchymal stem cell-derived microvesicles on articular cartilage defect. Transl Res. (2018) 193:42-53. doi: 10.1016/j.trsl.2017.12.003

22. Kim HS, Choi DY, Yun SJ, Choi SM, Kang JW, Jung JW, et al. Proteomic analysis of microvesicles derived from human mesenchymal stem cells. $J$ Proteome Res. (2012) 11:839-49. doi: 10.1021/pr200682z

23. Qiu G, Zheng G, Ge M, Wang J, Huang R, Shu Q, et al. Functional proteins of mesenchymal stem cell-derived extracellular vesicles. Stem Cell Res Ther. (2019) 10:359. doi: 10.1186/s13287-019-1484-6

24. Alcayaga-Miranda F, Cuenca J, Khoury M. Antimicrobial activity of mesenchymal stem cells: current status and new perspectives of antimicrobial peptide-based therapies. Front Immunol. (2017) 8:339. doi: 10.3389/fimmu.2017.00339

25. Wang XQ, Zhu XJ, Zou P. [Research progress of mesenchymal stem cellderived microvesicle]. Zhongguo Shi Yan Xue Ye Xue Za Zhi. (2013) 21:22730. doi: 10.7534/j.issn.1009-2137.2013.01.046

26. Collino F, Bruno S, Incarnato D, Dettori D, Neri F, Provero P, et al. AKI recovery induced by mesenchymal stromal cell-derived extracellular vesicles carrying microRNAs. J Am Soc Nephrol. (2015) 26:2349-60. doi: 10.1681/ASN.2014070710

27. Bruno S, Grange C, Collino F, Deregibus MC, Cantaluppi V, Biancone $\mathrm{L}$, et al. Microvesicles derived from mesenchymal stem cells enhance survival in a lethal model of acute kidney injury. PLoS ONE. (2012) 7:e33115. doi: 10.1371/journal.pone.0033115

28. Wang X, Gu H, Qin D, Yang L, Huang W, Essandoh K, et al. Exosomal miR-223 contributes to mesenchymal stem cell-elicited cardioprotection in polymicrobial sepsis. Sci Rep. (2015) 5:13721. doi: 10.1038/srep13721

29. Chen L, Lu FB, Chen DZ, Wu JL, Hu ED, Xu LM, et al. BMSCsderived miR-223-containing exosomes contribute to liver protection in experimental autoimmune hepatitis. Mol Immunol. (2018) 93:38-46. doi: 10.1016/j.molimm.2017.11.008
30. Xin H, Katakowski M, Wang F, Qian JY, Liu XS, Ali MM, et al. MicroRNA cluster miR-17-92 cluster in exosomes enhance neuroplasticity and functional recovery after stroke in rats. Stroke. (2017) 48:74753. doi: 10.1161/STROKEAHA.116.015204

31. Fonsato V, Collino F, Herrera MB, Cavallari C, Deregibus MC, Cisterna B, et al. Human liver stem cell-derived microvesicles inhibit hepatoma growth in SCID mice by delivering antitumor microRNAs. Stem Cells. (2012) 30:198598. doi: 10.1002/stem.1161

32. Ono M, Kosaka N, Tominaga N, Yoshioka Y, Takeshita F, Takahashi RU, et al. Exosomes from bone marrow mesenchymal stem cells contain a microRNA that promotes dormancy in metastatic breast cancer cells. Sci Signal. (2014) 7:ra63. doi: 10.1126/scisignal.2005231

33. Li J, Zhang Y, Liu Y, Dai X, Li W, Cai X, et al. Microvesicle-mediated transfer of microRNA-150 from monocytes to endothelial cells promotes angiogenesis. J Biol Chem. (2013) 288:23586-96. doi: 10.1074/jbc.M113.4 89302

34. Yu B, Gong M, Wang Y, Millard RW, Pasha Z, Yang Y, et al. Cardiomyocyte protection by GATA-4 gene engineered mesenchymal stem cells is partially mediated by translocation of miR-221 in microvesicles. PLoS ONE. (2013) 8:e73304. doi: 10.1371/journal.pone.0073304

35. Haraszti RA, Didiot MC, Sapp E, Leszyk J, Shaffer SA, Rockwell HE, et al. High-resolution proteomic and lipidomic analysis of exosomes and microvesicles from different cell sources. J Extracell Vesicles. (2016) 5:32570. doi: 10.3402/jev.v5.32570

36. Llorente A, Skotland T, Sylvanne T, Kauhanen D, Rog T, Orlowski A, et al Molecular lipidomics of exosomes released by PC-3 prostate cancer cells. Biochim Biophys Acta. (2013) 1831:1302-9. doi: 10.1016/j.bbalip.2013.04.011

37. Simbari F, McCaskill J, Coakley G, Millar M, Maizels RM, Fabrias G, et al. Plasmalogen enrichment in exosomes secreted by a nematode parasite versus those derived from its mouse host: implications for exosome stability and biology. J Extracell Vesicles. (2016) 5:30741. doi: 10.3402/jev.v5.30741

38. Tan KT, Lip GY. The potential role of platelet microparticles in atherosclerosis. Thromb Haemost. (2005) 94:488-92. doi: 10.1160/TH05-03-0201

39. Bruno S, Camussi G. Role of mesenchymal stem cell-derived microvesicles in tissue repair. Pediatr Nephrol. (2013) 28:224954. doi: 10.1007/s00467-013-2413-z

40. Alcayaga-Miranda F, Varas-Godoy M, Khoury M. Harnessing the angiogenic potential of stem cell-derived exosomes for vascular regeneration. Stem Cells Int. (2016) 2016:3409169. doi: 10.1155/2016/3409169

41. Park J, Kim S, Lim H, Liu A, Hu S, Lee J, et al. Therapeutic effects of human mesenchymal stem cell microvesicles in an ex vivo perfused human lung injured with severe E. coli pneumonia. Thorax. (2018) 74:4350. doi: 10.1136/thoraxjnl-2018-211576

42. Zhu YG, Feng XM, Abbott J, Fang XH, Hao Q, Monsel A, et al. Human mesenchymal stem cell microvesicles for treatment of Escherichia coli endotoxin-induced acute lung injury in mice. Stem Cells. (2014) 32:11625. doi: 10.1002/stem. 1504

43. Monsel A, Zhu YG, Gennai S, Hao Q, Hu S, Rouby JJ, et al. Therapeutic effects of human mesenchymal stem cell-derived microvesicles in severe pneumonia in mice. Am J Respir Crit Care Med. (2015) 192:32436. doi: 10.1164/rccm.201410-1765OC

44. Lee JW, Krasnodembskaya A, McKenna DH, Song Y, Abbott J, Matthay MA. Therapeutic effects of human mesenchymal stem cells in ex vivo human lungs injured with live bacteria. Am J Respir Crit Care Med. (2013) 187:75160. doi: 10.1164/rccm.201206-0990OC

45. Krasnodembskaya A, Samarani G, Song Y, Zhuo H, Su X, Lee JW, et al. Human mesenchymal stem cells reduce mortality and bacteremia in gramnegative sepsis in mice in part by enhancing the phagocytic activity of blood monocytes. Am J Physiol Lung Cell Mol Physiol. (2012) 302:L100313. doi: 10.1152/ajplung.00180.2011

46. Wang Y, Tan L, Jin J, Sun H, Chen Z, Tan X, et al. Non-cultured dermalderived mesenchymal cells attenuate sepsis induced by cecal ligation and puncture in mice. Sci Rep. (2015) 5:16973. doi: 10.1038/srep16973

47. Song Y, Dou H, Li X, Zhao X, Li Y, Liu D, et al. Exosomal miR146a contributes to the enhanced therapeutic efficacy of interleukin-1betaprimed mesenchymal stem cells against sepsis. Stem Cells. (2017) 35:120821. doi: 10.1002/stem.2564 
48. Tang XD, Shi L, Monsel A, Li XY, Zhu HL, Zhu YG, et al. Mesenchymal stem cell microvesicles attenuate acute lung injury in mice partly mediated by Ang-1 mRNA. Stem Cells. (2017) 35:1849-59. doi: 10.1002/stem. 2619

49. Phinney DG, Di Giuseppe M, Njah J, Sala E, Shiva S, St Croix CM, et al. Mesenchymal stem cells use extracellular vesicles to outsource mitophagy and shuttle microRNAs. Nat Commun. (2015) 6:8472. doi: 10.1038/ncomms9472

50. Zhang Z. Biomarkers, diagnosis and management of sepsis-induced acute kidney injury: a narrative review. Heart Lung Vessel. (2015) 7:64-73.

51. Gatti S, Bruno S, Deregibus MC, Sordi A, Cantaluppi V, Tetta C, et al. Microvesicles derived from human adult mesenchymal stem cells protect against ischaemia-reperfusion-induced acute and chronic kidney injury. Nephrol Dial Transplant. (2011) 26:1474-83. doi: 10.1093/ndt/gfr015

52. Zou X, Gu D, Xing X, Cheng Z, Gong D, Zhang G, et al. Human mesenchymal stromal cell-derived extracellular vesicles alleviate renal ischemic reperfusion injury and enhance angiogenesis in rats. Am J Transl Res. (2016) 8:4289-99.

53. Lin KC, Yip HK, Shao PL, Wu SC, Chen KH, Chen YT, et al. Combination of adipose-derived mesenchymal stem cells (ADMSC) and ADMSC-derived exosomes for protecting kidney from acute ischemia-reperfusion injury. Int $J$ Cardiol. (2016) 216:173-85. doi: 10.1016/j.ijcard.2016.04.061

54. Zhu G, Pei L, Lin F, Yin $\mathrm{H}$, Li X, He W, et al. Exosomes from human-bone-marrow-derived mesenchymal stem cells protect against renal ischemia/reperfusion injury via transferring miR-199a-3p. J Cell Physiol. (2019) 234:23736-749. doi: 10.1002/jcp.28941

55. Yuan X, Wang X, Chen C, Zhou J, Han M. Bone mesenchymal stem cells ameliorate ischemia/reperfusion-induced damage in renal epithelial cells via microRNA-223. Stem Cell Res Ther. (2017) 8:146. doi: 10.1186/s13287-017-0599-x

56. Tomasoni S, Longaretti L, Rota C, Morigi M, Conti S, Gotti E, et al. Transfer of growth factor receptor mRNA via exosomes unravels the regenerative effect of mesenchymal stem cells. Stem Cells Dev. (2013) 22:77280. doi: $10.1089 / \mathrm{scd} .2012 .0266$

57. Choi HY, Moon SJ, Ratliff BB, Ahn SH, Jung A, Lee M, et al. Microparticles from kidney-derived mesenchymal stem cells act as carriers of proangiogenic signals and contribute to recovery from acute kidney injury. PLOS ONE. (2014) 9:e87853. doi: 10.1371/journal.pone.0087853

58. Bruno S, Grange C, Deregibus MC, Calogero RA, Saviozzi S, Collino F, et al. Mesenchymal stem cell-derived microvesicles protect against acute tubular injury. J Am Soc Nephrol. (2009) 20:1053-67. doi: 10.1681/ASN.200 8070798

59. Reis LA, Borges FT, Simoes MJ, Borges AA, Sinigaglia-Coimbra R, Schor N. Bone marrow-derived mesenchymal stem cells repaired but did not prevent gentamicin-induced acute kidney injury through paracrine effects in rats. PLoS ONE. (2012) 7:e44092. doi: 10.1371/journal.pone.00 44092

60. Shen B, Liu J, Zhang F, Wang Y, Qin Y, Zhou Z, et al. CCR2 positive exosome released by mesenchymal stem cells suppresses macrophage functions and alleviates ischemia/reperfusion-induced renal injury. Stem Cells Int. (2016) 2016:1240301. doi: 10.1155/2016/1240301

61. Mendt M, Rezvani K, Shpall E. Mesenchymal stem cell-derived exosomes for clinical use. Bone Marrow Transplant. (2019) 54 (Suppl. 2):78992. doi: 10.1038/s41409-019-0616-z

62. Wang X, Huang W, Yang Y, Wang Y, Peng T, Chang J, et al. Loss of duplexmiR-223 (5p and 3p) aggravates myocardial depression and mortality in polymicrobial sepsis. Biochim Biophys Acta. (2014) 1842:70111. doi: 10.1016/j.bbadis.2014.01.012

63. Tabet F, Vickers KC, Cuesta Torres LF, Wiese CB, Shoucri BM, Lambert G, et al. HDL-transferred microRNA-223 regulates ICAM-1 expression in endothelial cells. Nat Commun. (2014) 5:3292. doi: 10.1038/ncomms4292

64. Feng Y, Huang W, Wani M, Yu X, Ashraf M. Ischemic preconditioning potentiates the protective effect of stem cells through secretion of exosomes by targeting Mecp2 via miR-22. PLoS One. (2014) 9:e88685. doi: 10.1371/journal.pone.0088685

65. Yu B, Kim HW, Gong M, Wang J, Millard RW, Wang Y, et al. Exosomes secreted from GATA-4 overexpressing mesenchymal stem cells serve as a reservoir of anti-apoptotic microRNAs for cardioprotection. Int J Cardiol. (2015) 182:349-60. doi: 10.1016/j.ijcard.2014.12.043
66. Lai RC, Yeo RW, Tan KH, Lim SK. Mesenchymal stem cell exosome ameliorates reperfusion injury through proteomic complementation. Regen Med. (2013) 8:197-209. doi: 10.2217/rme.13.4

67. Bian S, Zhang L, Duan L, Wang X, Min Y, Yu H. Extracellular vesicles derived from human bone marrow mesenchymal stem cells promote angiogenesis in a rat myocardial infarction model. J Mol Med. (2014) 92:38797. doi: 10.1007/s00109-013-1110-5

68. Wang $\mathrm{K}$, Jiang $\mathrm{Z}$, Webster KA, Chen J, Hu H, Zhou Y, et al. Enhanced cardioprotection by human endometrium mesenchymal stem cells driven by exosomal microRNA-21. Stem Cells Transl Med. (2017) 6:20922. doi: $10.5966 / \mathrm{sctm} .2015-0386$

69. Ma J, Zhao Y, Sun L, Sun X, Zhao X, Sun X, et al. Exosomes derived from Akt-modified human umbilical cord mesenchymal stem cells improve cardiac regeneration and promote angiogenesis via activating platelet-derived growth factor D. Stem Cells Transl Med. (2017) 6:51-9. doi: 10.5966/sctm.201 6-0038

70. Gonzalez-King H, Garcia NA, Ontoria-Oviedo I, Ciria M, Montero JA, Sepulveda P. Hypoxia inducible factor-1alpha potentiates jagged 1-mediated angiogenesis by mesenchymal stem cell-derived exosomes. Stem Cells. (2017) 35:1747-59. doi: 10.1002/stem.2618

71. Vrijsen KR, Maring JA, Chamuleau SA, Verhage V, Mol EA, Deddens JC, et al. Exosomes from cardiomyocyte progenitor cells and mesenchymal stem cells stimulate angiogenesis via EMMPRIN. Adv Healthc Mater. (2016) 5:255565. doi: $10.1002 / \mathrm{adhm} .201600308$

72. Wysoczynski M, Pathan A, Moore JBT, Farid T, Kim J, Nasr M, et al. Proangiogenic actions of CMC-derived extracellular vesicles rely on selective packaging of angiopoietin 1 and 2, but Not FGF-2 and VEGF. Stem Cell Rev Rep. (2019) 15:530-42. doi: 10.1007/s12015-019-09891-6

73. Xiao C, Wang K, Xu Y, Hu H, Zhang N, Wang Y, et al. Transplanted mesenchymal stem cells reduce autophagic flux in infarcted hearts via the exosomal transfer of miR-125b. Circ Res. (2018) 123:56478. doi: 10.1161/CIRCRESAHA.118.312758

74. Kramer L, Jordan B, Druml W, Bauer P, Metnitz PG. Incidence and prognosis of early hepatic dysfunction in critically ill patients-a prospective multicenter study. Crit Care Med. (2007) 35:1099-104. doi: 10.1097/01.CCM.0000259462.97164.A0

75. Chen G, Deng H, Song X, Lu M, Zhao L, Xia S, et al. Reactive oxygen species-responsive polymeric nanoparticles for alleviating sepsis-induced acute liver injury in mice. Biomaterials. (2017) 144:30-41. doi: 10.1016/j.biomaterials.2017.08.008

76. Nong K, Wang W, Niu X, Hu B, Ma C, Bai Y, et al. Hepatoprotective effect of exosomes from human-induced pluripotent stem cell-derived mesenchymal stromal cells against hepatic ischemia-reperfusion injury in rats. Cytotherapy. (2016) 18:1548-59. doi: 10.1016/j.jcyt.2016.08.002

77. Tamura R, Uemoto S, Tabata Y. Immunosuppressive effect of mesenchymal stem cell-derived exosomes on a concanavalin A-induced liver injury model. Inflamm Regen. (2016) 36:26. doi: 10.1186/s41232-016-0030-5

78. Haga H, Yan IK, Takahashi K, Matsuda A, Patel T. Extracellular vesicles from bone marrow-derived mesenchymal stem cells improve survival from lethal hepatic failure in mice. Stem Cells Transl Med. (2017) 6:126272. doi: $10.1002 / \mathrm{sctm} .16-0226$

79. Yan Y, Jiang W, Tan Y, Zou S, Zhang H, Mao F, et al. hucMSC exosome-derived GPX1 is required for the recovery of hepatic oxidant injury. Mol Ther. (2017) 25:465-79. doi: 10.1016/j.ymthe.2016.11.019

80. Tan CY, Lai RC, Wong W, Dan YY, Lim SK, Ho HK. Mesenchymal stem cellderived exosomes promote hepatic regeneration in drug-induced liver injury models. Stem Cell Res Ther. (2014) 5:76. doi: 10.1186/scrt465

81. Lai RC, Arslan F, Lee MM, Sze NS, Choo A, Chen TS, et al. Exosome secreted by MSC reduces myocardial ischemia/reperfusion injury. Stem Cell Res. (2010) 4:214-22. doi: 10.1016/j.scr.2009.12.003

82. Chen TS, Lai RC, Lee MM, Choo AB, Lee CN, Lim SK. Mesenchymal stem cell secretes microparticles enriched in pre-microRNAs. Nucleic Acids Res. (2010) 38:215-24. doi: 10.1093/nar/gkp857

83. Collino F, Deregibus MC, Bruno S, Sterpone L, Aghemo G, Viltono L, et al. Microvesicles derived from adult human bone marrow and tissue specific mesenchymal stem cells shuttle selected pattern of miRNAs. PLoS ONE. (2010) 5:e11803. doi: 10.1371/journal.pone.0011803 
84. Farsad K. Exosomes: novel organelles implicated in immunomodulation and apoptosis. Yale J Biol Med. (2002) 75:95-101.

85. Zhou YF, Bosch-Marce M, Okuyama H, Krishnamachary B, Kimura H, Zhang L, et al. Spontaneous transformation of cultured mouse bone marrow-derived stromal cells. Cancer Res. (2006) 66:10849-54. doi: 10.1158/0008-5472.CAN-06-2146

86. Buyanovskaya OA, Kuleshov NP, Nikitina VA, Voronina ES, Katosova LD, Bochkov NP. Spontaneous aneuploidy and clone formation in adipose tissue stem cells during different periods of culturing. Bull Exp Biol Med. (2009) 148:109-12. doi: 10.1007/s10517-009-0647-3

87. Kordelas L, Rebmann V, Ludwig AK, Radtke S, Ruesing J, Doeppner TR, et al. MSC-derived exosomes: a novel tool to treat therapy-refractory graft-versushost disease. Leukemia. (2014) 28:970-3. doi: 10.1038/leu.2014.41

88. Bruno S, Collino F, Deregibus MC, Grange C, Tetta C, Camussi G. Microvesicles derived from human bone marrow mesenchymal stem cells inhibit tumor growth. Stem Cells Dev. (2013) 22:75871. doi: $10.1089 /$ scd. 2012.0304
89. Mortaza S, Martinez MC, Baron-Menguy C, Burban M, de la Bourdonnaye M, Fizanne L, et al. Detrimental hemodynamic and inflammatory effects of microparticles originating from septic rats. Crit Care Med. (2009) 37:2045-50. doi: 10.1097/CCM.0b013e318 $1 \mathrm{a} 00629$

Conflict of Interest: The authors declare that the research was conducted in the absence of any commercial or financial relationships that could be construed as a potential conflict of interest.

Copyright (c) 2020 Cheng, Cao and Qin. This is an open-access article distributed under the terms of the Creative Commons Attribution License (CC BY). The use, distribution or reproduction in other forums is permitted, provided the original author(s) and the copyright owner(s) are credited and that the original publication in this journal is cited, in accordance with accepted academic practice. No use, distribution or reproduction is permitted which does not comply with these terms. 